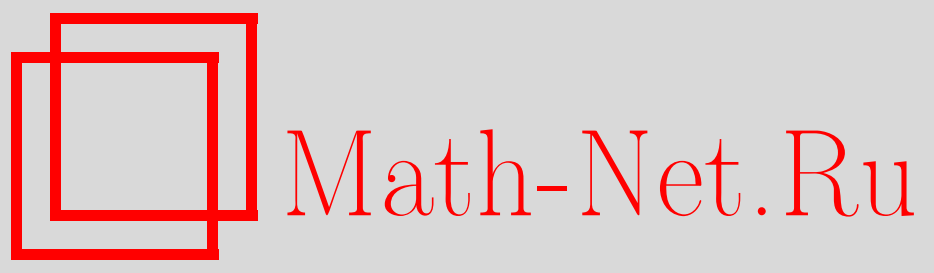

С. Ю. Славянов, Д. А. Шатько, А. М. Ишханян, Т. А. Ротинян, Генерация и удаление ложных особенностей в линейных обыкновенных дифференциальных уравнениях с полиномиальными коэффициентами, ТМФ, 2016, том 189, номер 3, 371-379

DOI: https://doi.org/10.4213/tmf9229

Использование Общероссийского математического портала Math-Net.Ru подразумевает, что вы прочитали и согласны с пользовательским соглашением http: //www . mathnet.ru/rus/agreement

Параметры загрузки:

IP : 54.81 .137 .203

26 апреля 2023 г., $17: 21: 27$

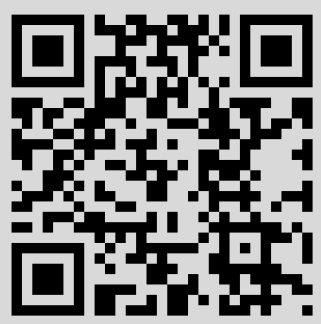




\title{
ГЕНЕРАЦИЯ И УДАЛЕНИЕ ЛОЖНЫХ ОСОБЕННОСТЕЙ В ЛИНЕЙНЫХ ОБЫКНОВЕННЫХ ДИФФЕРЕНЦИАЛЬНЫХ УРАВНЕНИЯХ С ПОЛИНОМИАЛЬНЫМИ КОЭФФИЦИЕНТАМИ
}

\begin{abstract}
Рассмотрены примеры генерации ложных особых точек при дифференцировании частных однородных линейных обыкновенных дифференциальных уравнений с полиномиальными коэффициентами. Сформулированы две гипотезы общего характера, касающиеся генерации и удаления ложных особых точек в произвольных дифференциальных уравнениях фуксового типа с полиномиальными коэффициентами. Обсуждается модельная задача из физики полимеров.
\end{abstract}

Ключевые слова: уравнения класса Гойна, уравнения Пенлеве, ложные особые точки.

DOI: $10.5062 / \operatorname{tmf} 9229$

\section{1. ВВЕДЕНИЕ}

За последние годы исследованию специфических свойств обыкновенных дифференциальных уравнений с ложными особыми точками был посвящен ряд публикаций (см., например, работы [1]-[5]). В частности, в статье [1] выявлена важная роль таких особых точек при получении шестерки уравнений Пенлеве из уравнений Гойна. В работах [4], [5] уравнения с ложными особыми точками используются для построения решений уравнений Гойна в виде разложений в ряды по обыкновенным и обобщенным гипергеометрическим функциям.

Работа А. М. Ишханяна поддержана Государственным комитетом по науке Министерства образования и науки Республики Армения (грант № 15T-1C323), а также проектом "Ведущие исследовательские университеты России" (грант FTI_24_2016 Томского политехнического университета).

* Санкт-Петербургский государственный университет, Санкт-Петербург, Россия. E-mail: slav@ss2034.spb.edu

${ }^{\dagger}$ Институт физических исследований НАН Армении, Аштарак-2, Армения

‡Физико-технический институт Томского политехнического университета, Томск, Россия 
Среди обыкновенных линейных дифференциальных уравнений можно выделить уравнения с полиномиальными коэффициентами, т.е. такие, где перед производными зависимой переменной стоят полиномы от независимой (комплексной) переменной. Нули полинома при старшей производной, а также в общем случае точка являются сингулярными точками (сингулярностями, особыми точками) данного уравнения. Чтобы выяснить, является ли бесконечность сингулярностью, можно применить преобразование Мёбиуса и перевести точку $z=\infty$ в конечную область комплексной z-плоскости.

Если решения линейного дифференциального уравнения в окрестности некоторой конечной сингулярности являются функциями ограниченного роста, т. е. растут не быстрее определенной степени расстояния до сингулярности, то сингулярность является фуксовой. Уравнения, все сингулярности которых фуксовы, включая бесконечность, называются фуксовыми уравнениями. Заметим, что имеются фуксовы уравнения, которые не имеют вида уравнений с полиномиальными коэффициентами, однако сводятся к таковым калибровочными преобразованиями. С другой стороны, к фуксовым уравнениям с полиномиальными коэффициентами калибровочные преобразования неприменимы, так как нарушают полиномиальность коэффициентов.

В настоящей статье нами показано, что в обыкновенных линейных дифференциальных уравнениях с полиномиальными коэффициентами ложные особые точки можно породить посредством дифференцирования уравнений, не содержащих таких особенностей, и наоборот, интегрируя уравнения с ложными особенностями, можно оттуда их удалить.

Наше исследование базируется на рассмотрении обыкновенных дифференциальных уравнений с полиномиальными коэффициентами, в которых полином наивысшей степени стоит при наивысшего порядка производной от зависимой переменной. Вдобавок мы полагаем, что степень этого полинома превышает порядок дифференциального уравнения. Таким образом, рассматривается уравнение вида

$$
\sum_{k=0}^{n} P_{k}(z) \frac{d^{n-k} w(z)}{d z^{n-k}}=0
$$

в котором $P_{0}(z)$ является полиномом наивысшей степени среди всех $P_{k}(z)$, причем степень $m$ этого полинома превосходит порядок $n$ данного дифференциального уравнения: $m>n$. Наконец, мы полагаем, что нули полинома $P_{0}(z)$ и точка $z=\infty$ являются (ввиду некоторых дополнительных не обсуждаемых здесь ограничений) регулярными особыми точками, так что уравнение (1) оказывается уравнением из ряда фуксовых.

При обсуждении некоторых частных примеров таких фуксовых уравнений мы показываем, что если полином $P_{n}(z)$ при последнем члене суммы в уравнении (1), а именно коэффициент при $w(z)$, принимает нулевое значение в конечной точке $q$ комплексной плоскости, то в уравнении, которому удовлетворяет производная от решения уравнения (1), в общем случае в той же самой точке $q$ появляется дополнительная ложная особая точка. На основе данного наблюдения мы формулируем две гипотезы общего свойства, касающиеся генерации (удаления) ложных особых точек в (из) произвольных фуксовых дифференциальных уравнениях (уравнений) с полиномиальными коэффициентами. 
Далее мы рассматриваем конфлюэнтные модификации уравнения (1) с нерегулярными особыми точками. Заметим, что нерегулярные особые точки образуются при слиянии регулярных особых точек в результате определенного процесса их коалесценции. В этом случае коэффициенты получившегося в итоге уравнения остаются полиномами; однако чередование степеней последовательных полиномов в сумме (1) вообще перестает быть монотонным. Мы показываем, что генерировать и удалять ложные особые точки посредством соответственно дифференцирования и интегрирования удается и в случае конфлюэнтных уравнений. В качестве примера для иллюстрации взята частная модель из физики полимеров.

\section{2. ПРИМЕРЫ}

ПримеР 1. Рассмотрим уравнение Гойна, имеющее три фуксовы сингулярности в конечных точках $z_{j}, j=1,2,3$, и на бесконечности (без ограничения общности считаем, что $\left.z_{1}=0, z_{2}=1, z_{3}=t\right)$ :

$$
\begin{gathered}
P_{0}(z) w^{\prime \prime}(z)+P_{1}(z) w^{\prime}(z)+P_{2}(z) w(z)=0, \\
P_{0}(z)=\prod_{j=1}^{3}\left(z-z_{j}\right), \\
P_{1}(z)=\sum_{k=1}^{3}\left(1-\theta_{k}\right) \frac{P_{0}(z)}{z-z_{k}}, \\
P_{2}(z)=\alpha \theta_{\infty}(z-q),
\end{gathered}
$$

причем выполняется тождество Фукса

$$
\sum_{k=1}^{3} \theta_{k}+\theta_{\infty}+\alpha=2 .
$$

Обобщенный символ Римана (см. книгу [6]) для этого уравнения имеет вид

$$
\left(\begin{array}{ccccc}
z_{1} & z_{2} & z_{3} & \infty & z \\
0 & 0 & 0 & \alpha & q \\
\theta_{1} & \theta_{2} & \theta_{3} & \theta_{\infty} &
\end{array}\right)
$$

Продифференцируем уравнение $(2)$ по $z$, обозначим $u(z)=w^{\prime}(z)$ и исключим $w(z)$ из получившегося уравнения. Тогда получаем уравнение

$$
\begin{aligned}
P_{0}(z) u^{\prime \prime}(z) & +\left(P_{1}(z)+P_{0}^{\prime}(z)-P_{0}(z) \frac{1}{z-q}\right) u^{\prime}(z)+ \\
& +\left(P_{2}(z)+P_{1}^{\prime}(z)-P_{1}(z) \frac{1}{z-q}\right) u(z)=0 .
\end{aligned}
$$

Это уравнение, которое мы назовем деформированным уравнением Гойна, помимо сингулярностей, присутствующих в исходном уравнении Гойна (2), в общем случае имеет дополнительную ложную особую точку $z=q$. Дополнительная особая точка появляется, если $q$ - корень полинома $P_{2}(z)$ - не является корнем полинома $P_{0}(z)[4]$. Заметим, что то, что степень полинома $P_{2}(z)$ равна единице, является 
ключевым условием для наших построений (случай степени более высокого порядка рассмотрен ниже). Нулевая степень, имеющая место для гипергеометрического уравнения, не приводит к появлению ложной особой точки. Тем не менее в литературе уравнения гипергеометрического класса с добавленной особой точкой также рассматриваются [3]. Такая ситуация обусловлена тем, что проводятся дополнительные преобразования исходного уравнения.

Умножив уравнение (8) на $z-q$, получаем фуксово уравнение с полиномиальными коэффициентами, но уже с еще одной конечной фуксовой особой точкой, причем новый полином при $u(z)$ является полиномом второго порядка. Заметим, что в общем случае $z=q$ не является корнем этого полинома. Продифференцировав уравнение (8) еще раз, получаем уравнение, вообе говоря, уже с двумя ложными особыми точками. Наоборот, проинтегрировав уравнение, придем к уравнению без ложной особой точки.

Завершая данный пример, заметим, что вместо линейных фуксовых дифференциальных уравнений второго порядка часто рассматривают фуксовы $(2 \times 2)$-системы линейных уравнений первого порядка, которые можно свести к эквивалентным фуксовым уравнениям второго порядка, причем возникающие уравнения содержат ложные особые точки [7], [8].

Пример 2. Рассмотрим вновь уравнение второго порядка типа (2), но с числом конечных фуксовых особых точек, равным $m$, так что

$$
\begin{aligned}
& P_{0}(z)=\prod_{j=1}^{m}\left(z-z_{j}\right) \\
& P_{1}(z)=\sum_{k=1}^{m}\left(1-\theta_{k}\right) \frac{P_{0}(z)}{z-z_{k}}, \\
& P_{2}(z)=\alpha \theta_{\infty} \prod_{j=1}^{m-2}\left(z-q_{j}\right) .
\end{aligned}
$$

В рассматриваемом случае полином $P_{2}(z)$ является полиномом степени $m-2$, причем тождество Фукса принимает вид

$$
\sum_{k=1}^{m} \theta_{k}+\theta_{\infty}+\alpha=m-1 .
$$

Продифференцируем уравнение (2) по $z$. Тогда для производной $u(z)=w^{\prime}(z)$ приходим к уравнению

$$
\begin{aligned}
P_{0}(z) u^{\prime \prime}(z) & +\left(P_{1}(z)+P_{0}^{\prime}(z)-P_{0}(z)\left[\sum_{j=1}^{m-2} \frac{1}{z-q_{j}}\right]\right) u^{\prime}(z)+ \\
& +\left(P_{2}(z)+P_{1}^{\prime}(z)-P_{1}(z)\left[\sum_{j=1}^{m-2} \frac{1}{z-q_{j}}\right]\right) u(z)=0 .
\end{aligned}
$$

В общем случае, когда все параметры $q_{j}$ различны (т. е. полином $P_{2}(z)$ не имеет кратных корней) и не являются корнями полинома $P_{0}(z)$, у этого уравнения по 
сравнению с исходным уравнением имеется $m-2$ дополнительных ложных особых точки (для каждой из этих точек разность между характеристическими показателями равна двум).

ПримеР 3. Пусть в предыдущем примере верны равенства $m=4$ и $q_{1}=q_{2}=q$, т. е. $P_{2}(z)=\alpha \theta_{\infty}(z-q)^{2}$, так что точка $z=q$ является нулем кратности два. Тогда, проведя те же действия, мы вновь придем к ложной сингулярности, но несколько иной, чем рассмотренные выше. Действительно, в этом случае

$$
\frac{P_{2}^{\prime}(z)}{P_{2}(z)}=\frac{2}{z-q}
$$

так что для производной $u(z)=w^{\prime}(z)$ деформированное уравнение имеет следующий вид:

$$
P_{0}(z) u^{\prime \prime}(z)+\left(P_{1}(z)+P_{0}^{\prime}(z)-\frac{2}{z-q} P_{0}(z)\right) u^{\prime}(z)+\left(P_{2}(z)+P_{1}^{\prime}(z)-\frac{2}{z-q} P_{1}(z)\right) u(z)=0 .
$$

Соответственно разность характеристических показателей в точке $z=q$ равна не двум, как в предыдущих случаях, а трем. Это означает, что одно линейно независимое решение в точке $z=q$ имеет нулевую третью производную, а разложение другого решения в степенной ряд начинается с третьей степени.

Точно по такой же схеме можно получать ложные особые точки с разностью характеристических показателей, равной четырем, пяти и т. д.

ПримеР 4. Рассмотрим особое фуксово уравнение третьего порядка с конечными регулярными сингулярностями, расположенными в точках $z_{1}=0, z_{2}=1, z_{3}=t$ :

$$
\begin{aligned}
z^{2}(z-1)(z-t) w^{\prime \prime \prime}(z) & +\left[(3-\alpha-\beta) z(z-1)(z-t)-\theta_{2} z^{2}(z-t)-\theta_{3} z^{2}(z-1)\right] w^{\prime \prime}(z)+ \\
+ & (\alpha-1)(\beta-1)(z-1)(z-t) w^{\prime}(z)+\kappa(z-q) w(z)=0 .
\end{aligned}
$$

Обобщенный символ Римана, задающий характеристические показатели в сингулярностях, для этого уравнения имеет вид

$$
\left(\begin{array}{ccccc}
z_{1} & z_{2} & z_{3} & \infty & z \\
0 & 0 & 0 & a & q \\
\alpha & 1 & 1 & b & \\
\beta & 2+\theta_{2} & 2+\theta_{3} & c &
\end{array}\right)
$$

Характеристические показатели, входящие в этот символ, свидетельствуют о том, что в особой точке $z=0$ есть одно голоморфное решение и два решения, которые, вообще говоря, не являются голоморфными. В то же время в окрестностях точек $z=1$ и $z=t$ имеются два линейно независимых голоморфных решения и одно решение, которое не является голоморфным. Можно указать определенные соотношения между характеристическими показателями в особой точке $z=\infty$ и параметрами $\alpha$, $\beta, \kappa, \theta_{2}, \theta_{3}$. Эти соотношения имеют вид

$$
\begin{gathered}
a+b+c=-\alpha-\beta-\theta_{2}-\theta_{3}, \\
a b+b c+a c=\alpha \beta+\theta_{2}+\theta_{3}, \\
a b c=\kappa .
\end{gathered}
$$


Продифференцировав уравнение (16), получаем уравнение третьего порядка для $u(z)=w^{\prime}(z)$ с ложной особой точкой $z=q$

$$
u^{\prime \prime \prime}+\Delta_{1}(z) u^{\prime \prime}+\Delta_{2}(z) u^{\prime}+\Delta_{3}(z) u=0,
$$

где введены обозначения

$$
\begin{gathered}
\Delta_{1}(z)=\frac{5-\alpha-\beta}{z}-\frac{\theta_{2}-1}{z-1}-\frac{\theta_{3}-1}{z-t}-\frac{1}{z-q} \\
\Delta_{2}(z)=\frac{(2-\alpha)(2-\beta)}{z^{2}}+\frac{3-\alpha-\beta}{z} \Sigma(z)-\frac{2 \theta_{2}}{z(z-1)}-\frac{2 \theta_{3}}{z(z-t)}- \\
-\frac{\theta_{2}+\theta_{3}}{(z-1)(z-t)}+\frac{\theta_{2}}{(z-q)(z-1)}+\frac{\theta_{3}}{(z-q)(z-t)} \\
\Delta_{3}(z)=\frac{(\alpha-1)(\beta-1)}{z^{2}} \Sigma(z)+\frac{\kappa(z-q)}{z^{2}(z-1)(z-t)} \\
\Sigma(z)=\frac{1}{z-1}+\frac{1}{z-t}-\frac{1}{z-q}
\end{gathered}
$$

Это уравнение можно считать деформированным уравнением по отношению к (16).

\section{3. ГИПОТЕЗЫ}

Рассмотренные примеры позволяют сформулировать следующие гипотезы.

ГиПотеЗА 1. Любое фуксово уравнение с полиномиальными коэффициентами, имеющее ложные сингулярности, можно получить как соответствующую производную от фуксова уравнения с полиномиальными коэффициентами без ложных сингулярностей.

При этом в ходе выкладок допускается спецификация параметров. Если воспользоваться обратным дифференцированием, то приходим к противоположной гипотезе.

ГиПотЕзА 2. Любое фуксово уравнение с полиномиальными коэффициентами, имеющее ложные сингулярности, можно преобразовать к фуксовому уравнению с полиномиальными коэффициентами без ложных сингулярностей с использованием обратного дифферениирования (интегрирования).

Доказательство этих гипотез - открытый вопрос. Основной проблемой является конструирование общего конкретизированного представления для фуксова уравнения с полиномиальными коэффициентами.

\section{4. КОНФЛЮЭНТНЫЕ УРАВНЕНИЯ}

Для конфлюэнтных уравнений с полиномиальными коэффициентами также применим механизм генерации ложных фуксовых особых точек за счет дифференцирования уравнений. Однако здесь возникает существенно большее разнообразие случаев, требующих рассмотрения. Проблема состоит в том, что описание даже формального вида решения в окрестности нерегулярной особой точки авторам настоящей статьи неизвестно, как неизвестен и характер чередования степеней полиномов. Поэтому мы ограничимся здесь обсуждением лишь наиболее простого, но важного для приложений вида уравнений, а именно уравнений класса Гойна. 
Конфлюэнтные уравнения класса Гойна - это линейные дифференциальные уравнения второго порядка, которые можно представить в том же виде, что и уравнение Гойна

$$
P_{0}(z) w^{\prime \prime}(z)+P_{1}(z) w^{\prime}(z)+P_{2}(z) w(z)=0,
$$

но с другими по сравнению с уравнением (2) правилами образования степеней входящих полиномов. Мы ограничимся здесь только нередуцированными уравнениями, т. е. такими, где все степенные ряды в окрестностях сингулярностей содержат лишь целые степени.

Для них полином $P_{0}(z)$ является полиномом второй степени или меньше, полином $P_{1}(z)$ является полиномом второй степени, но, что является кардинально важным, полином $P_{2}(z)$ есть полином первой степени с одним нулем (например, в точке $z=q$ ). Все полиномы зависят от параметра $t$, который переходит из одного уравнения в другое при конфлюэнции.

Введем производную $u(z)=w^{\prime}(z)$. Дифференцирование уравнения (26) приводит к уравнению для $u(z)$, которое формально совпадает с уравнением $(8)$ :

$P_{0}(z) u^{\prime \prime}(z)+\left(P_{1}(z)+P_{0}^{\prime}(z)-P_{0}(z) \frac{P_{2}^{\prime}(z)}{P_{2}(z)}\right) u^{\prime}(z)+\left(P_{2}(z)+P_{1}^{\prime}(z)-P_{1}(z) \frac{P_{2}^{\prime}(z)}{P_{2}(z)}\right) u(z)=0$.

Поскольку для всех четырех конфлюэнтных уравнений Гойна справедливо соотношение $P_{2}(z)=\alpha(z-q)$, это уравнение помимо сингулярностей в исходном уравнении класса Гойна имеет дополнительную ложную сингулярность в точке $z=q$. Естественно, данный результат имеет место с оговоркой, что точка $z=q$ не совпадает с уже существующими сингулярностями; иными словами, ложная сингулярность появляется, если $q$ не является корнем полинома $P_{0}(z)[9]$.

\section{5. ОДНА ФИЗИЧЕСКАЯ МОДЕЛЬ}

Обратимся к одной модели в физике полимеров. Оценка времени релаксации полимерной молекулы, находящейся в плоском гидродинамическом растягивающем потоке, связана с решением следующей спектральной задачи [10]:

$z(z-1) w^{\prime \prime}(z)+\left(-\kappa z(z-1)+\frac{3}{2}(z-1)+(b+1) z\right) w^{\prime}(z)+((\nu-\kappa)(z-1)-2 b \kappa z) w(z)=0$,

где

$$
w(0)=0, \quad w(1)=0 .
$$

Параметр $b$ называется гибкостью. Он принимает большие значения $\left(b \sim 10^{2}-10^{4}\right)$ и устанавливает размеры области вблизи фуксовой сингулярности $z=1$, где функция $w(z)$ пренебрежимо мала. Параметр $\kappa$ выражается как $\kappa=b W$, где величина $W$ называется числом Вайсенберга. Это число описывает уровень растяжения. Согласно физическим представлениям критическое значение $W=1 / 2$ отмечает переход из состояния свернутости в состояние растянутости в растягивающих потоках. При $W<1 / 2$ полимеры свернуты; при $W>1 / 2$, т. е. если $W$ превышает критическое значение, полимеры целиком развернуты. Наконец, величину $\nu$ можно рассматривать как собственное значение, и именно первое собственное значение $\nu_{1}$ дает оценку 
времени релаксации

$$
T_{\mathrm{rel}}=\frac{b \tau}{\nu_{1}}
$$

где $\tau$ - время релаксации полимера к равновесию с окружающей средой. Поставленная задача неоднократно исследовалась численно. Здесь в соответствии с темой настоящей статьи мы приводим уравнение для производной $u(z)=w^{\prime}(z)$. Введем положение ложной сингулярности

$$
z=q=\frac{\nu-\kappa}{\nu-\kappa-2 b \kappa} .
$$

Тогда

$$
\begin{gathered}
z(z-1) u^{\prime \prime}(z)+\left(-\kappa z(z-1)+\frac{3(z-1)}{2}+(b+1) z+2 z-1-\frac{z(z-1)}{z-q}\right) u^{\prime}(z)+ \\
+\left((\nu-\kappa)(z-1)-2 b \kappa z-\kappa(2 z-1)+\left(\frac{5}{2}+b\right)-\right. \\
\left.-\frac{-\kappa z(z-1)+3(z-1) / 2+(b+1) z}{z-q}\right) u(z)=0 .
\end{gathered}
$$

Далее полученное уравнение можно использовать по рецепту, рассмотренному в статье [1], для вывода динамического нелинейного уравнения.

\section{6. ОБСУЖДЕНИЕ}

Специфическим свойством ложной сингулярности, делающей ее заметной, является следующее. К любой обычной точке дифференциального уравнения приложимы условия Коши; тогда все другие производные можно рассчитать, исходя из самого дифференциального уравнения. В случае ложной сингулярности условия Коши по-прежнему применимы, однако производная второго или высшего порядка может оказаться произвольной.

Поведение решений обыкновенных линейных дифференциальных уравнений в окрестности фуксовой особой точки характеризуется фробениусовскими показателями в этой точке. Для уравнений с полиномиальными коэффициентами один из этих показателей в любой конечной точке оказывается равным нулю. Решение с таким показателем является голоморфным в окрестности указанной сингулярности. Возникает вопрос, существуют ли другие голоморфные решения. Сингулярности, для которых такие решения существуют, называются ложными. Необходимым условием для того, чтобы сингулярность можно было назвать ложной, является то, чтобы разность между двумя характеристическими показателями оказалась целым числом больше единицы.

В настоящей статье на основе нескольких представительных примеров изучены аналогии и различия между уравнениями, содержащими и не содержащими ложные особые точки. Продемонстрировано, что в результате дифференцирования уравнений без ложных особых точек последние могут возникать в дифференциальных уравнениях с полиномиальными коэффициентами, как фуксовых, так и конфлюэнтных. И наоборот, интегрированием уравнений ложные сингулярности можно удалять. Выдвинута гипотеза, что для фуксовых уравнений с полиномиальными коэффициентами указанные свойства носят всеобщий характер. 
Нам хотелось бы завершить исследование замечанием, что использование ложных особых точек способно стимулировать развитие нескольких новых полезных разработок. В частности, оно позволяет по-новому взглянуть на задачу Римана-Гильберта.

\section{Список литературы}

[1] С. Ю. Славянов, О. Л. Стесик, ТМФ, 186:1 (2016), 142-151.

[2] A. V. Shanin, R. V. Craster, European J. Appl. Math., 13:6 (2002), 617-639.

[3] A. Ya. Kazakov, Internat. J. Theor. Math. Phys., 3:6 (2013), 190-196.

[4] A. Ishkhanyan, K.-A. Suominen, J. Phys. A: Math. Gen., 36:5 (2003), L81-L85.

[5] C. Leroy, A. M. Ishkhanyan, Integral Transforms Spec. Func., 26:6 (2015), 451-459.

[6] С. Ю. Славянов, В. Лай, Специальные функиии: единая теория, основанная на анализе особенностей, Невский диалект, СПб., 2002.

[7] А. Я. Казаков, С. Ю. Славянов, ТМФ, 155:2 (2008), 252-264.

[8] С. Ю. Славянов, ТМФ, 182:2 (2015), 223-230.

[9] V. A. Shahnazaryan, T. A. Ishkhanyan, T. A. Shahverdyan, A. M. Ishkhanyan, Armenian J. Phys., 5:3 (2012), 146-155.

[10] D. Vincenzi, E. Bodenschatz, J. Phys. A: Math. Gen., 39:34 (2006), 10691-10701.

Поступила в редакцию 13.05.2016 